\title{
Toward scalable fabrication of electrochemical paper sensor without surface functionalization
}

\author{
Cheng Wang ${ }^{1}$, Rui $\mathrm{Wu}^{1}$, Hao Ling ${ }^{1}$, Zhiling Zhao $\mathbb{D}^{2}$, Wenjia Han ${ }^{3}$, Xiaowen Shi ${ }^{4}$, Gregory F. Payne $\mathbb{D}^{2}$ and Xiaohui Wang $\mathbb{D}^{1 凶}$
}

Paper-based electrochemical sensors provide the opportunity for low-cost, portable and environmentally friendly single-use chemical analysis and there are various reports of surface-functionalized paper electrodes. Here we report a composite paper electrode that is fabricated through designed papermaking using cellulose, carbon fibers (CF), and graphene oxide (GO). The composite paper has well-controlled structure, stable, and repeatable properties, and offers the electrocatalytic activities for sensitive and selective chemical detection. We demonstrate that this CF/GO/cellulose composite paper can be reduced electroche mically using relatively mild conditions and this $\mathrm{GO}$ reduction confers electrocatalytic properties to the composite paper. Finally, we demonstrate that this composite paper offers sensing performance (sensitivity and selectivity) comparable to, or better than, paperbased sensors prepared by small-batch surface-modification (e.g., printing) methods. We envision this coupling of industrialized papermaking technologies with interfacial engineering and electrochemical reduction can provide a platform for single-use and portable chemical detection for a wide range of applications.

npj Flexible Electronics (2022)6:12; https://doi.org/10.1038/s41528-022-00143-1

\section{INTRODUCTION}

Cellulose-based paper has attracted considerable attention as a matrix for portable single-use electrochemical sensing because of its low cost, lightweight, and biodegradability ${ }^{1-7}$. Typical routes for generating paper-based electrochemical sensors use post functionalization methods (e.g., printing) to deposit components that confer conductivity and electrocatalytic activity onto the surface of the paper substrate ${ }^{8-13}$. These studies have demonstrated impressive performance attributes and suggest an exciting vision forward, however, a key requirement toward the widespread adoption of paper-based electrochemical sensors will be the scalable fabrication of stable, low-cost paper-based electrodes with repeatable properties, which still remains a challenge.

The challenges for advancing the practical application of paperbased electrochemical sensor lie in two aspects: first, the performance of reported paper-based sensors varies in a great extent due to the diversity in substrate features (e.g., porosity, network, and surface), complicated process, and strict conditions in small-batch production, leading to the key concerns for uncertainty in reproducibility and accuracy ${ }^{14-16}$; second, the surface of paper-based sensors must be specifically designed and modified to meet the requirement for selective detection, and the performance of the paper-based material cannot be controlled on a large scale ${ }^{17-19}$. Taking screen printing as an example, many factors, including the viscosity and solid content of printing ink, printing strength, and the structure of paper substrate, all affect the sensitivity of paper-based sensor leading to great uncertainty in repeatability. These challenges can hardly be resolved, unless standard paper electrode with well-controlled structure and imbed functionality can be scalably produced and directly used needing no surface treatment. However, it is difficult to integrate functional materials with paper into defined structure and area in mass production because of the lack of bonding and interaction between cellulose fibers and functional materials.

Here, we realized the scalable production and controlled functionalization of paper, and report the use of this paper for sensitive and selective electrochemical detection based on two findings. First, we adapt conventional papermaking methods to generate a functional composite paper composed of cellulose fibers, carbon fibers (CF), and graphene oxide (GO). As illustrated in Fig. 1a, the successful fabrication of this composite required interfacial engineering to control the interactions between the hydrophilic cellulose fibers, hydrophobic CF, and nanosized GO fillers. Consequently, stable and uniform composite paper with repeatable properties was obtained, which could be further papercut into a large number of flexible electrodes with designed patterns. Then, Fig. $1 \mathrm{~b}$ shows that we used a comparatively mild and efficient electrochemical treatment $(<10 \mathrm{~min},<1 \mathrm{~V})$ to reduce the $\mathrm{GO}$ in the composite into its reduced form ( $\mathrm{rGO}$ ) to create function. As suggested, the CF confers conducting properties that facilitate the reduction of GO to enhance its electrocatalytic activity. Figure 1c suggests that this composite paper can provide a means to realize the vision of low-cost paper-based electrodes for electrochemical detection.

\section{RESULTS \\ Preparation and characterization of composite paper electrode}

A critical requirement for using papermaking technology to prepare stable and uniform composite paper with repeatable properties, is that the cellulose, CF, and GO must be uniformly dispersed in water to make pulp (Fig. 2a). The cellulose fibers and GO are hydrophilic with large negative charges, while the CF fibers are hydrophobic and lightweight with a small negative charge, as

\footnotetext{
${ }^{1}$ State Key Laboratory of Pulp and Paper Engineering, South China University of Technology (SCUT), Guangzhou 510640, China. ${ }^{2}$ Institute for Bioscience and Biotechnology Research (IBBR), University of Maryland, College Park, MD 20742, USA. ${ }^{3}$ State Key Laboratory of Biobased Material and Green Papermaking, Shandong Academy of Sciences, Qilu University of Technology, Jinan 250353, China. ${ }^{4}$ School of Resource and Environmental Science, Hubei Biomass-Resource Chemistry and Environmental Biotechnology Key Laboratory, Wuhan University, Wuhan 430079, China. ${ }^{\circledR}$ email: fewangxh@scut.edu.cn
} 
(a)

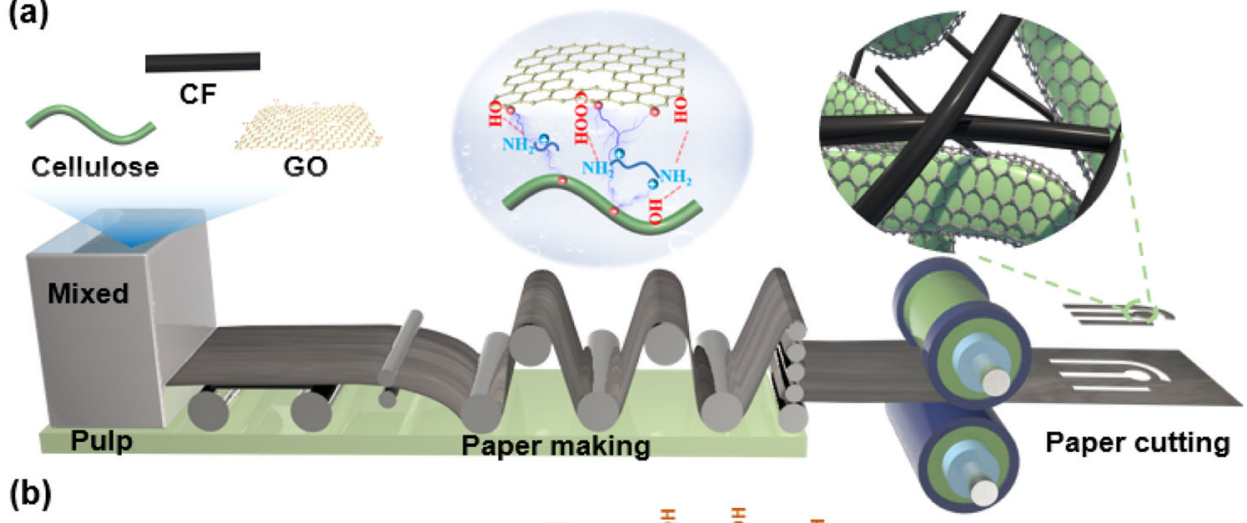

(b)
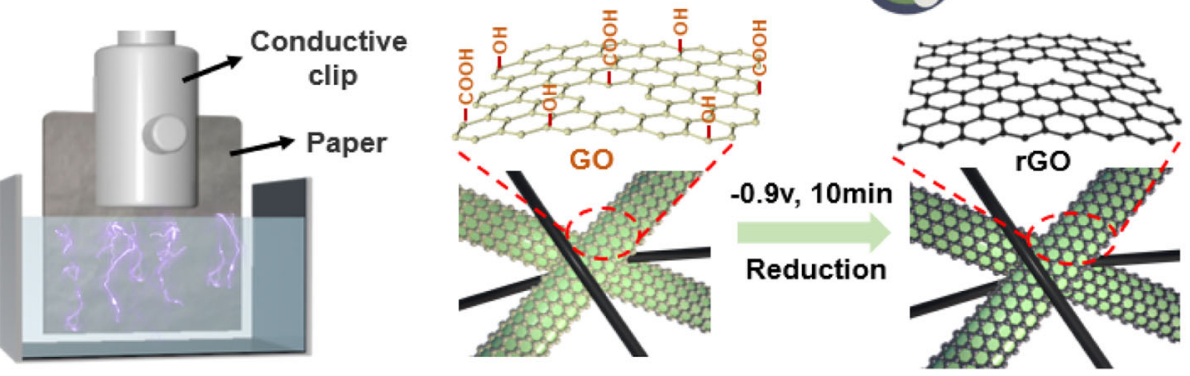

(c)
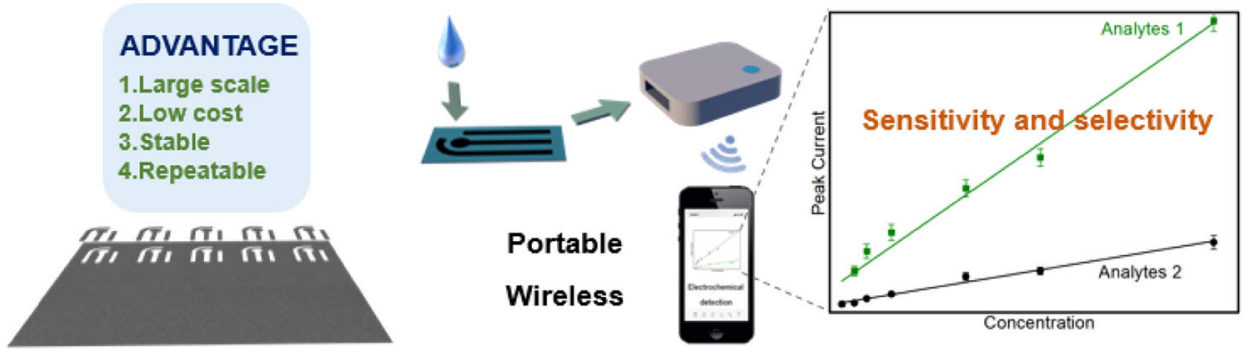

Fig. 1 Large-scale preparation of high-performance paper-based electrochemical sensors. a Large-scale papermaking. By controlling interfacial interactions, composite paper was prepared composed of cellulose, carbon fibers (CF, confers conductivity) and graphene oxidize (GO, confers electrocatalytic activities). b Electrochemistry reduction of GO. Electrochemistry provides a simple scalable approach to GO reduction. c Vision of paper-based electrochemical sensor. The vision for high-performance and sustainable paper-based sensors for portable electrochemical detection.

determined by the zeta-potential measurements in Fig. $2 b^{20}$. The diversity in the properties of fibers increases the difficulty of pulping and following papermaking. An interface-engineering approach was employed to address this problem. The series of photographs in Fig. 2a indicate that the addition of a small amount of cationic polyacrylamide (CPAM) promotes the association and aggregation of cellulose and GO, while the low-density and hydrophobic CF could be well dispersed within these GO/ cellulose aggregates. The resulting CF/GO/cellulose "pulp" could then be processed into composite paper and a very fast processing time is confirmed (further details of the pulping and processing steps are provided in Supplementary Fig. 1 of Supporting Information). By studying the electrochemical sensing and mechanical properties of different raw material ratios, the optimal ratio of CF/cellulose/GO was determined as 1/1/0.17 (further details of the electrochemical sensing and mechanical properties are provided in Supplementary Fig. 2 and Supplementary Fig. 3 of Supporting Information).

We employed three independent measurements to provide molecular and microstructural evidence for the effective integration of components into the composite paper. First, we use energy dispersive $\mathrm{X}$-ray spectroscopy (EDS) to provide evidence for the location of the CPAM (as measured by elemental nitrogen) in the composite paper. The addition of
CPAM to CF/GO/cellulose led to a significantly increased $\mathrm{N}$-content (compared with the $\mathrm{CF} /$ cellulose control without CPAM addition: Supplementary Fig. 4 of Supporting Information), and Fig. 2c shows that this $\mathrm{N}$-containing component was uniformly assembled onto the surface of the cellulose fiber. Second, we used micro-computed tomography (micro-CT) to examine the distribution of the carbon and cellulose fibers within the CF/GO/cellulose composite (note: micro-CT can discern these different fibers based on their different densities). Figure $2 d$ shows that the two fiber networks are uniformly distributed and interpenetrating throughout the network. Third, we used scanning electron microscopy (SEM) to observe the microstructure of the composite paper. Figure 2 e shows that $\mathrm{GO} /$ cellulose interweaves with CF to form a uniform network structure and GO sheets can be observed on the surface of cellulose fibers (further analysis is provided in Supplementary Fig. 5d of Supporting Information). The above results show that we have successfully prepared uniform and stable CF/GO/cellulose paper.

\section{Electrochemical reduction of GO}

The electrocatalytic activity of $\mathrm{GO}$ is often improved by reduction, and a variety of methods have been used to convert GO into its reduced $\mathrm{rGO}$ form ${ }^{21,22}$. As illustrated in Fig. 3a, we used a comparatively mild electrochemical method in which the 
(a)

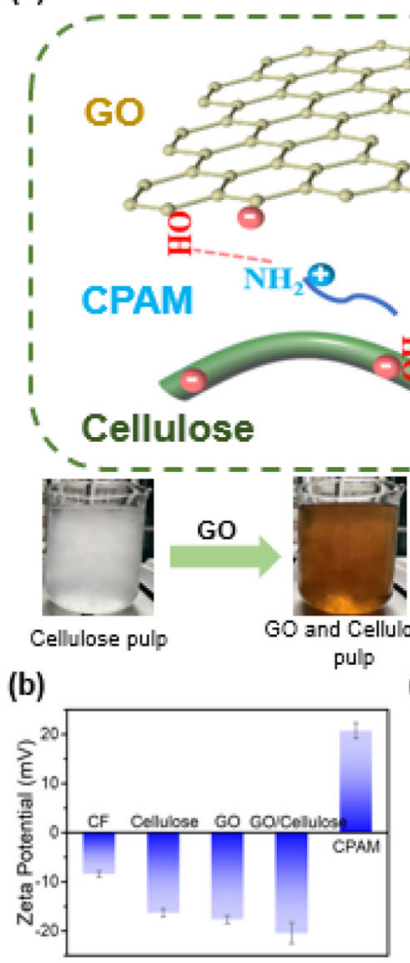

(d)

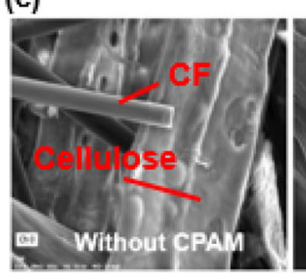

(e)
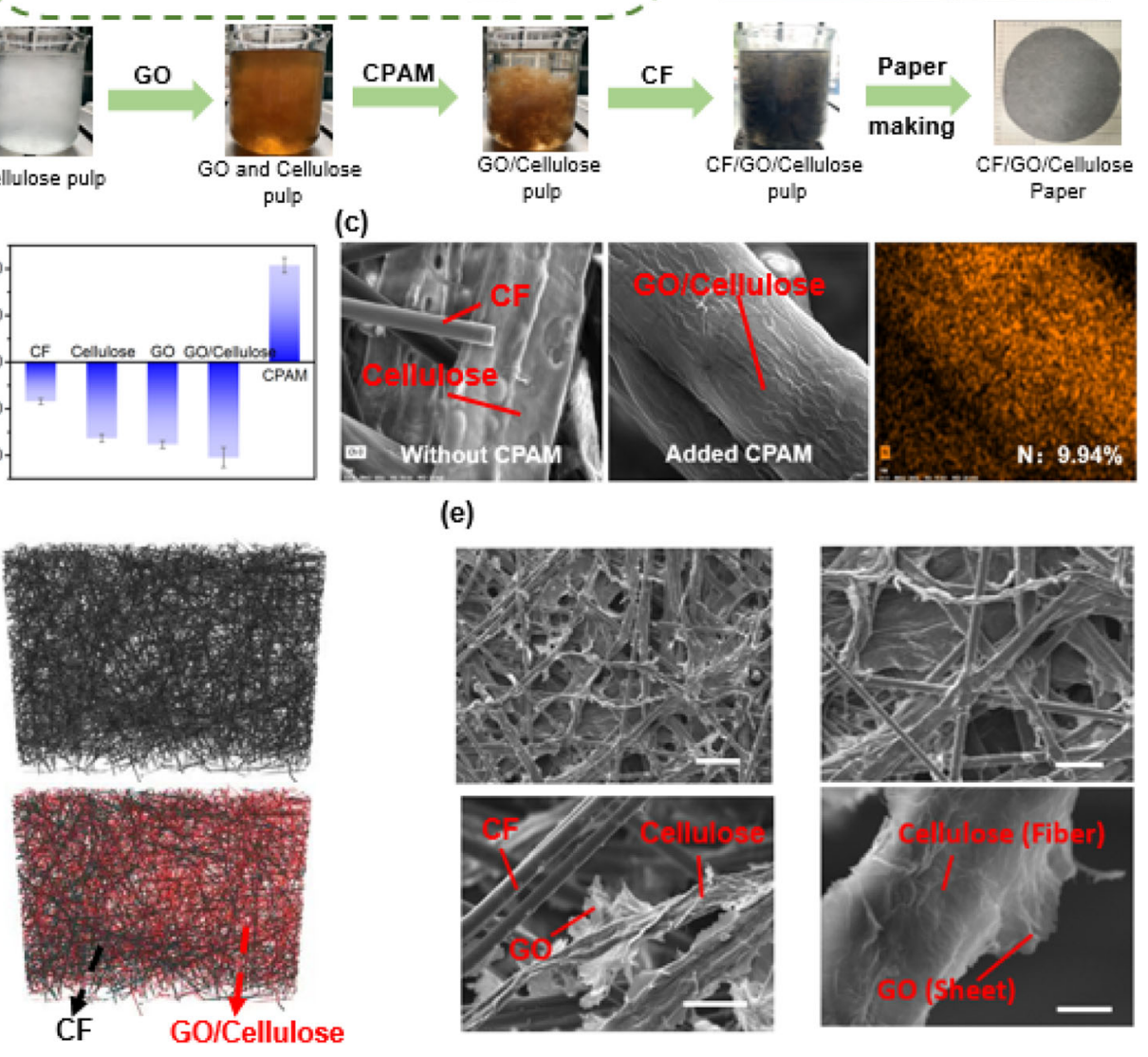

Fig. 2 Preparation and characterization of CF/GO/cellulose paper. a Fabrication of CF/GO/cellulose paper. Cationic polyacrylamide (CPAM) was used to control the interfacial interactions to enable the assembly of $\mathrm{GO}$ sheets onto the surface of the cellulose fibers. $\mathbf{b}$ Electrostatic interaction. Zeta-potential measurements indicate the electrostatic charge of the components. c The distribution of CPAM was detected by EDS. EDS shows that CPAM (determined by nitrogen) is localization on the surface of cellulose. $\mathbf{d}$ 3D structure by micro-CT. Micro-CT shows a homogeneous distribution of the CF and cellulose fibers in the CF/GO/cellulose composite. e Surface morphology by SEM. Scanning electron microscopy images show the composite's homogeneous distribution and also the assembly of the GO sheets on the cellulose surface. Scale bar is: top left $50 \mu \mathrm{m}$, bottom left $10 \mu \mathrm{m}$, top right $10 \mu \mathrm{m}$, and bottom right $1 \mu \mathrm{m}$.

composite paper was immersed in an electrolyte solution $(1 \mathrm{M} \mathrm{KCl}$, $\mathrm{pH} 7)$ and a cathodic voltage $(-0.9 \mathrm{~V}$ vs the $\mathrm{Ag} / \mathrm{AgCl}$ reference electrode) was applied. Figure $3 b$ shows current-time $(i-t)$ curves for the current drawn by several composite paper formulations. The leftmost plot shows that a control GO/cellulose (lacking CF) draws little current that demonstrates the importance of the carbon-fiber network for conferring conductivity to the composite. This plot also shows little current drawn for the CF/cellulose control (lacking GO), which suggests the current is being drawn to reduce the $\mathrm{GO}$ component. The rightmost plot in Fig. 3b shows the $i-t$ curves for CF/GO/cellulose composites prepared with differing amounts of GO. The inset in this plot indicates a nearly linear increase in electron transfer (charge; $Q=\int$ idt) with $\mathrm{GO}$ content. These electrochemical results indicate that the electrons transferred are reducing the GO component of the composite paper (additional electrochemical evidence for this conclusion is provided in Supplementary Fig. 6 of the Supporting Information).

Two chemical methods were used to provide molecular evidence for electrochemical GO-reduction. First, Raman spectroscopy was used as the $D$ and $G$ peaks in the Raman spectra are often correlated to graphene structure and the ratio of D- to G-peak intensity is often correlated to structural defects that are believed to be important to electrocatalytic activities ${ }^{23,24}$. The Raman spectra and summary bar chart in Fig. 3c show the results for CF/GO/cellulose composites that had been electrochemically reduced for various times along with the results from controls. This bar chart shows that electrochemical reduction led to an increase on the D- to G-peak ratio for the composite. Second, X-ray 
(a)

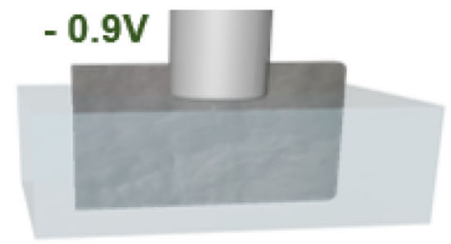

(b)

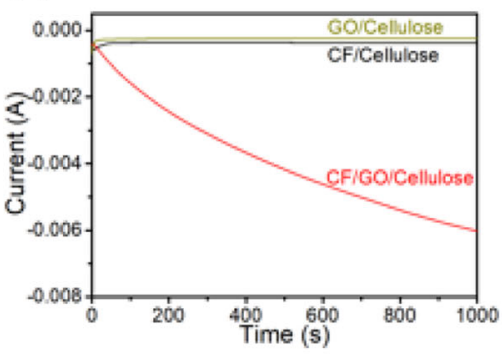

(c)

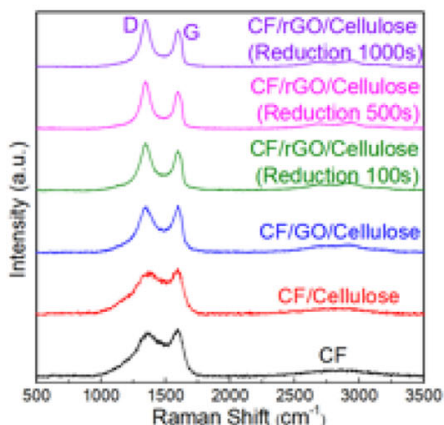

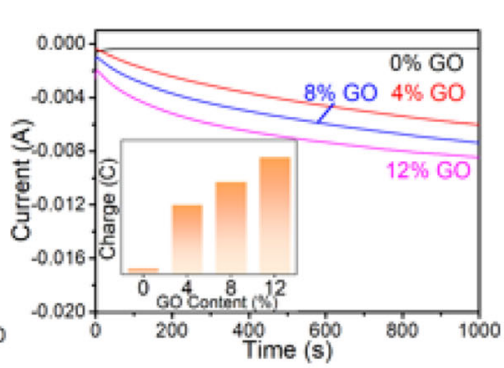

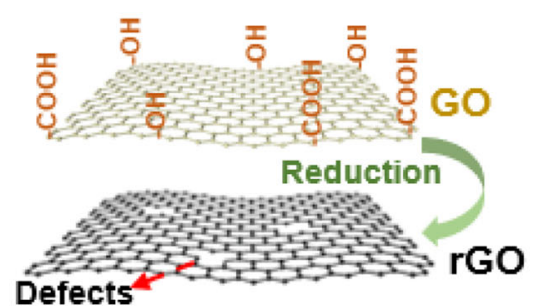

Defects

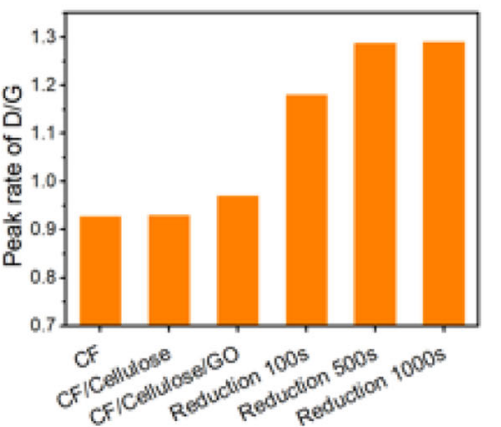

(d)
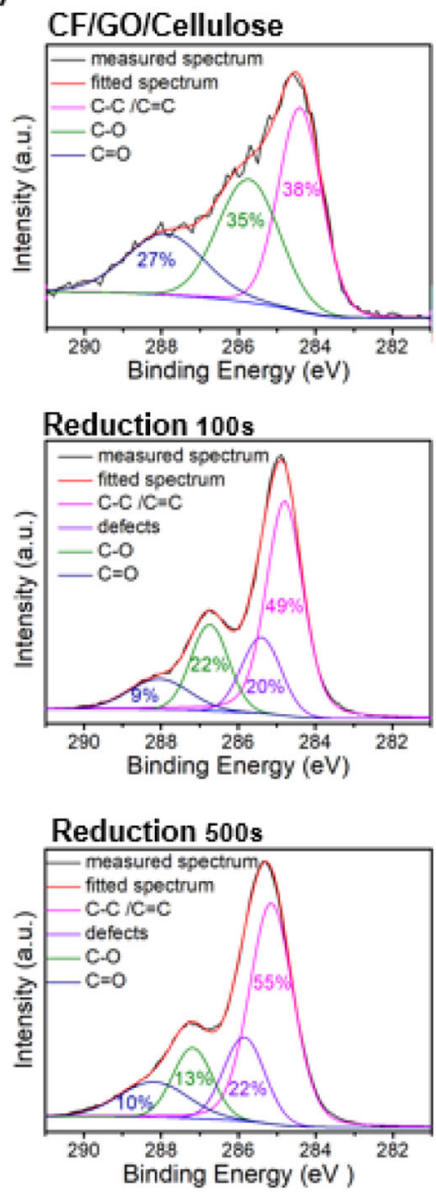

Fig. 3 Electrochemical reduction of $\mathbf{G O}$ in the composite paper. a Electrochemical reduction of graphene oxide. Schematic of electrochemical conversion of $\mathrm{GO}$ to reduced $\mathrm{GO}(\mathrm{rGO})$. b Controllable electrochemical reduction of $\mathrm{GO}$ in paper. Electrochemical measurements of current vs time (i-t) show that CF is required for reduction (CF provides the conducting network) and the reducing charge transferred $\left(Q=\int \mathrm{idt}\right)$ increases with $\mathrm{GO}$ content (GO is the species being reduced). c Raman evidence on $\mathrm{GO}$ electrochemical reduction. Raman spectroscopy provides molecular evidence for GO reduction and the generation of defects (indicated by $D$ band) is believed to be important for electrocatalytic activity. d High-resolution XPS spectra of C $1 \mathrm{~s}$. The high-resolution C $1 \mathrm{~s}$ peaks of XPS provide further molecular evidence for electrochemical reduction.

photoelectron spectroscopy (XPS) was used and the C $1 \mathrm{~s}$ region of the spectra was analyzed for chemical modification. The spectra in Fig. 3d show that electrochemical reduction resulted in decreases in the peaks for $\mathrm{C} / \mathrm{O}$ single and double bonds consistent with expectations. These chemical and electrochemical measurements both indicate that the $\mathrm{CF} / \mathrm{GO} /$ cellulose composite paper can be reduced electrochemically.

\section{Flexibility and stability of composite paper electrode}

To illustrate the macroscopic electrical properties of our composite paper, we used conventional cutting operations to create patterned "sensors" (Fig. 4a). The photographs in Fig. 4b provide a simple illustration that these composites possess conducting properties. We measured the sheet resistance of composite paper $\left(9 \Omega \mathrm{sq}^{-1}\right)$ and calculated its conductivity as $1389 \mathrm{Sm}^{-1}$. The schematic in Fig. 4c shows that these composites could also serve as working electrodes in a three-electrode electrochemical cell. In this experiment, the composite paper was tested using a standard electrochemical mediator potassium ferricyanide $\left(\mathrm{K}_{3} \mathrm{Fe}(\mathrm{CN})_{6}\right)$ in cyclic-voltammetry (CV) experiments. As illustrated, the CV curves were similar for electrodes that had been folded at different angles and even cut. These illustrations provide initial evidence for the electrochemical properties of the composite paper.
One functionally important feature of this composite paper is its stability in water. To illustrate this water stability, Fig. $4 \mathrm{~d}$ shows we immersed the composite electrode in water and after 60 hours, this composite paper showed no evidence of swelling or leaching of the GO components. To quantify this stability, we removed this composite paper from the water every 12 hours and tested it by $\mathrm{CV}$ using the $\mathrm{K}_{3} \mathrm{Fe}(\mathrm{CN})_{6}(5 \mathrm{mM})$. The bar chart of Fig. $4 \mathrm{~d}$ shows that the oxidation-peak current in this CV remains nearly constant over this 60 hour experiment. Another functionally important feature that results from the fabrication method is repeatability. To illustrate repeatability, we cut several sensors from different locations of the composite paper and we tested each cut sensor by performing $\mathrm{CV}$ measurements with the $\mathrm{K}_{3} \mathrm{Fe}(\mathrm{CN})_{6}$. The bar graph in Fig. 4e shows that the oxidation-peak current for these five different electrodes was similar (relative standard deviation among these five electrodes is less than $3.5 \%$ ). These results indicate that the composite paper offers important practical features for paper-based electrochemical sensing.

\section{Electrochemical sensing of phenols}

While CF confers conductivity to the composite paper, the reduced GO ( $\mathrm{GGO}$ ) confers electrocatalytic activities (additional electrochemical evidence for this conclusion is provided in 
(a)

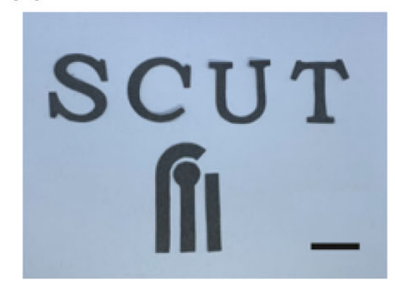

(b)

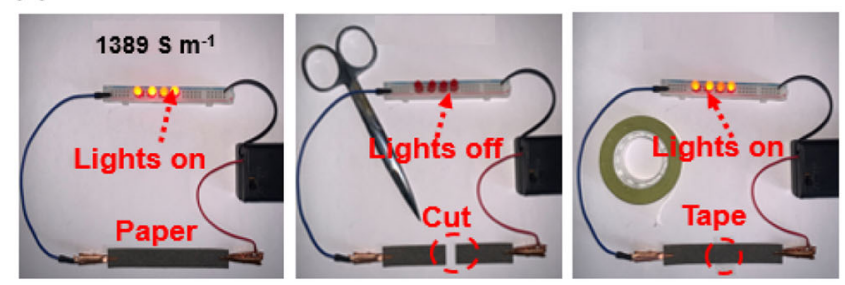

(c)

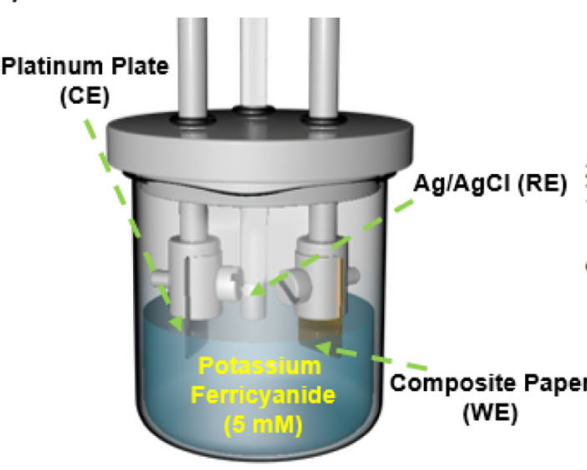

(d)

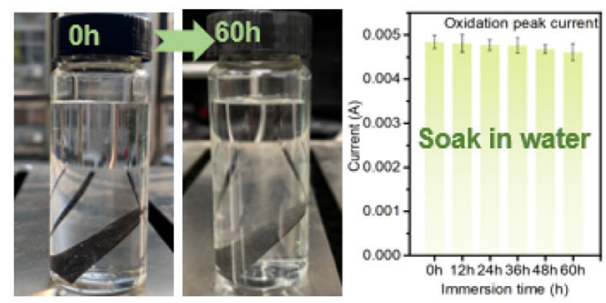

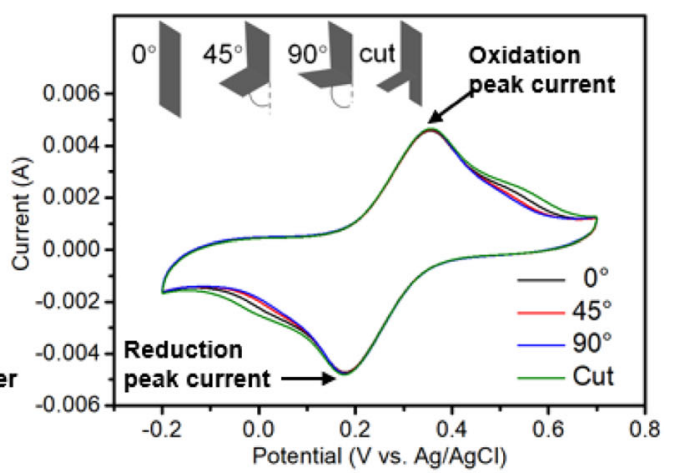

(e)

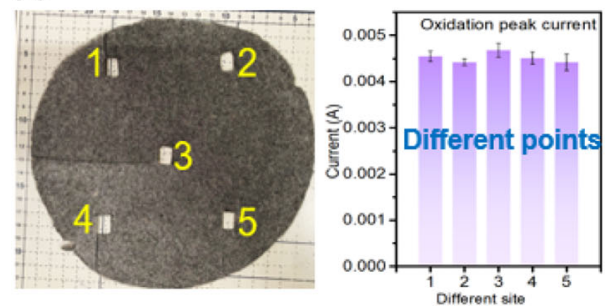

Fig. 4 Important features of CF/GO/cellulose composite paper. a Cut paper. Paper cutting allows reproducible generation of different patterns. Scale bar is $2 \mathrm{~cm}$. b Repairability. The composite paper is conducting (conductivity value is $1389 \mathrm{Sm}^{-1}$ ). Electrochemical measurements (of $\mathrm{K}_{3} \mathrm{Fe}(\mathrm{CN})^{6}$ ) demonstrate that the $\mathrm{CF} / \mathrm{GO} /$ cellulose composite is (c) flexibility (CV of potassium ferricyanide). Electrochemically active and flexible. d Stability. Structurally and functionally stable in water, and (e) repeatability. Repeatable in that different samples (i.e., different sensors) taken from different locations show the same functional performance.

Supplementary Fig. 7 and Supplementary Fig. 8 of the Supporting Information). To illustrate the importance of $\mathrm{GO}$ reduction on the composite's electrocatalytic activity, we prepared sensors that had been reduced to different extents by varying the electrochemical reduction time (and therefore the reductive charge transfer). As illustrated in Fig. 5a, these sensors were evaluated by performing $\mathrm{CV}$ measurements with $\mathrm{K}_{3} \mathrm{Fe}(\mathrm{CN})_{6}$. Sensors prepared from a CF/ $\mathrm{GO} / c e l l u l o s e$ composite that were not electrochemically reduced show no peaks for oxidation or reduction. Sensors prepared from composites that were electrochemically reduced show peak currents for $\mathrm{K}_{3} \mathrm{Fe}(\mathrm{CN})_{6}$ and these peak currents were larger for composites prepared with increasing reduction times ${ }^{25}$. The bar chart summarizes these results and demonstrates the importance of $\mathrm{GO}$ reduction for the composite's electrocatalytic activity.

As a proof-of-concept demonstration, we considered the analysis of three phenols that are commonly present in the environment ${ }^{26,27}$. As illustrated in Fig. 5b, hydroquinone (HQ) can be oxidized under mildly oxidative voltages, while $\mathrm{p}$-chlorophenol (CP) and p-nitrophenol (NP) require more positive voltages for their oxidation. For this demonstration study, we prepared composite paper from CF $(45.9 \% \mathrm{w} / \mathrm{w}), \mathrm{GO}(8 \% \mathrm{w} / \mathrm{w})$, cellulose $(45.9 \% \mathrm{w} / \mathrm{w})$, and CPAM $(0.2 \% \mathrm{w} / \mathrm{w})$, electrochemically reduced this composite $(-0.9 \mathrm{~V}, 10 \mathrm{~min})$, and then cut rectangular sensors (surface area $1 \mathrm{~cm}^{2}$ ). Electrochemical measurements were performed using a commonly used method of differential pulse voltammetry (DPV). When solutions containing a single phenol $\left(10 \mathrm{mg} \mathrm{L}^{-1}\right)$ were measured, the DPV curves in Fig. $5 \mathrm{~b}$ show that an oxidation peak was observed, and these oxidation peaks occurred at different voltages. When a solution was prepared containing all three phenols ( $10 \mathrm{mg} \mathrm{L}^{-1}$ for each phenol), Fig. $5 \mathrm{c}$ shows that three separate oxidation peaks were observed and the voltages where these peaks appeared are consistent with those for the individual phenols. The DPV curve for a control sensor prepared from a composite that was not electrochemically reduced shows no discernable oxidation peaks when tested with the mixture containing all three phenols. These results illustrate that the reduced graphene oxide $(\mathrm{rGO})$ confers electrocatalytic properties to the composite paper: electrocatalytic properties are generally believed to improve sensitivity by amplifying detection currents and improve selectivity by separating peaks to avoid interference ${ }^{28-30}$.

To test the detection sensitivity of the composite paper sensors, we analyzed solutions containing three phenols all present at the same concentration. The DPV curves in Fig. $5 \mathrm{c}$ show that oxidation peaks are observed for all three phenols and each of the peak currents was larger for solutions prepared with higher phenol concentrations. Each of these peak currents was quantified and the correlations between peak current and concentration are also shown in Fig. $5 \mathrm{c}$. The estimated detection limits of $\mathrm{HQ}, \mathrm{CP}$, and NP were $0.045,0.093$, and $0.571 \mathrm{mg} \mathrm{L}^{-1}$, respectively. The results from this demonstration study indicate that the composite paper offers appropriate electrocatalytic activities to serve as an electrochemical sensor. 
(a)

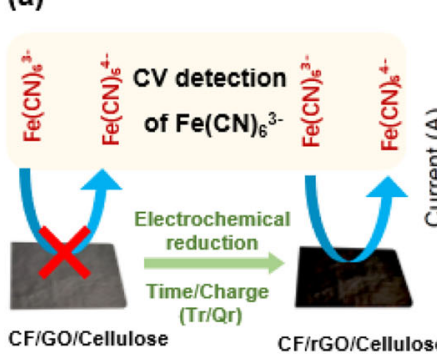

(b)
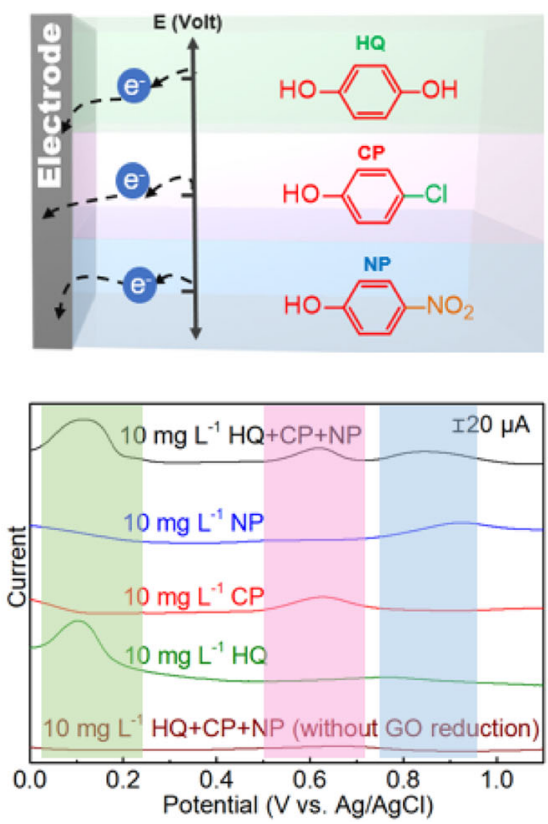

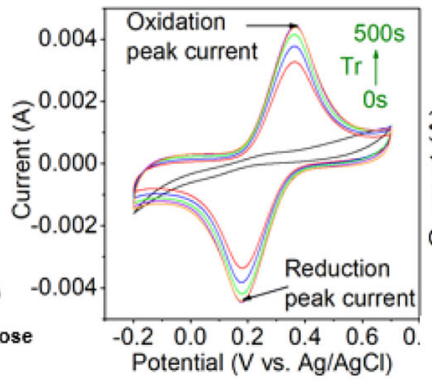

(c)
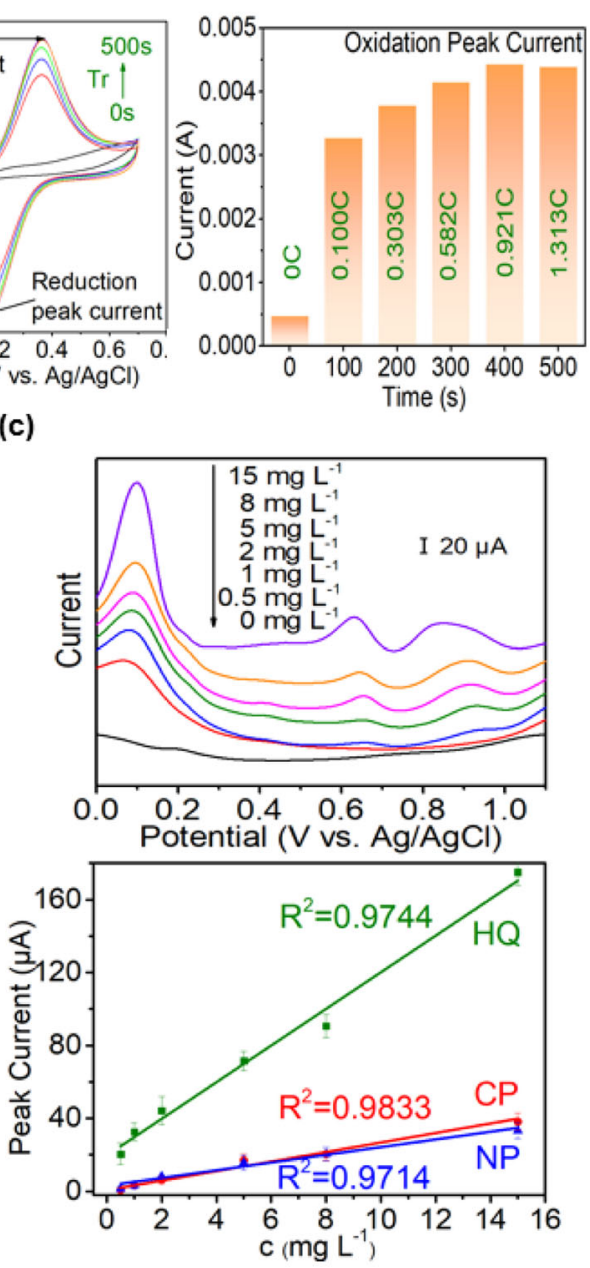

Fig. 5 Electrochemical detection by composite paper. a Electrocatalytic activities. Electrochemical reduction of CF/GO/cellulose confers electrocatalytic activity to the composite (as measured by CV measurements of $\mathrm{Fe}(\mathrm{CN})_{6}{ }^{3-}$ ). Differential pulse voltammetry (DPV) measurements with three phenols (hydroquinone, chlorophenol, and nitrophenol) illustrate that the electrocatalytic properties enhance (b) selectivity of electrochemical sensing. Detection selectivity (DPV-peak separation) and (c) sensitivity of electrochemical sensing. Detection sensitivity (peak amplification; error bars show the standard deviation from three measurements and are generally small relative to symbol size).

\section{Comprehensive evaluation}

We prepared the radar chart in Fig. 6 to provide a multivariate comparison of our composite paper with other representative electrochemical sensors that have been considered for hydroquinone $(\mathrm{HQ})$ analysis (the specific scoring criteria and values are provided in the Supporting Information). The two metrics at the upper left (limit of detection and $\mathrm{R}^{2}$ ) are quantitative values that characterize sensor performance, and these metrics show that our composite paper is comparable to other reported electrochemical sensors. For the ease of operation metric at the lower left, the two paper-based electrodes and screen-printed carbon electrodes (SPCE) were scored more highly because they would be disposed after a single use, while the glassy-carbon electrodes (GCE) would need to be reused (GCE's cost is relatively high). For the cost metric at the lower right, the composite paper was more favorable because sensors made from ball-milled graphene or gold incur significant additional raw material costs, and SPCE requires a high cost of substrate and conductive ink. Finally, the large-scale processability metric at the upper right shows a higher score for the composite paper as we envision the use of scalable-paper manufacturing and electrochemical-processing technologies for the fabrication of this composite. In contrast, GCE is more difficult to manufacture at a large scale, while surface modification with nanoparticles (gold or graphene) will likely require slower serial manufacturing steps (e.g., printing). Thus, as indicated in Fig. 6, we envision that our composite paper provides a low-cost and scalable approach to generate single-use electrodes with the necessary performance to realize the vision for portable electrochemical analysis.

\section{DISCUSSION}

We report the fabrication of a low-cost composite paper that offers conducting and electrocatalytic properties appropriate for electrochemical sensing. In contrast to other paper-based sensors prepared by surface modification (i.e., printing), the composite is prepared using conventional papermaking technologies and thus offers advantages in terms of repeatability and scalability. We believe that there are three important features of this work. First, controlling interfacial interactions is essential for generating a composite from equal quantities of the cellulose and carbon fibers, and also for assembling the GO sheets onto the cellulose fibers. Second, reduction of the GO confers electrocatalytic activities to the composite and we performed this reduction using an electrochemical step that is mild $(<1 \mathrm{~V}$ for several minutes) and scalable. Third, the composite paper has several 


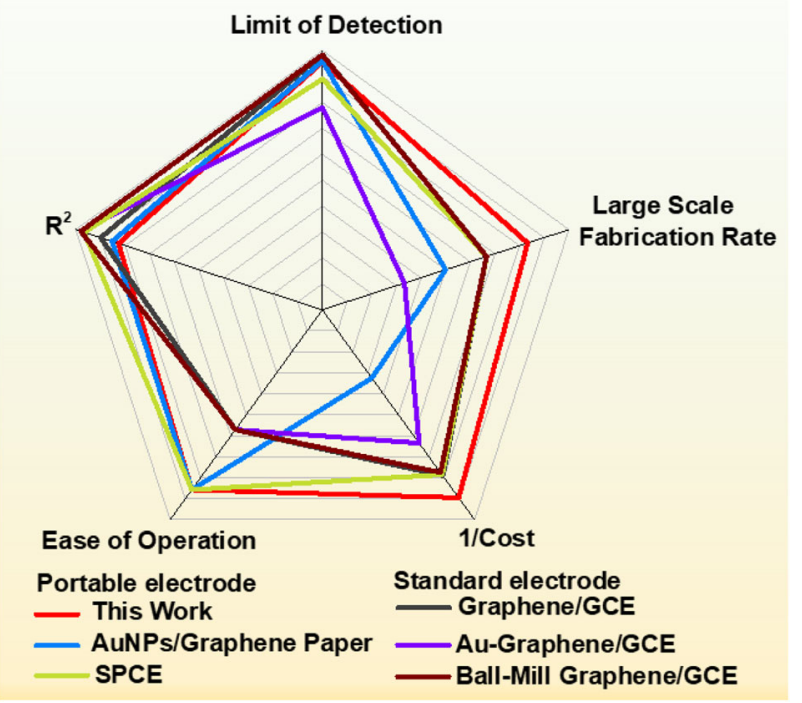

Fig. 6 Comparison of CF/GO/Cellulose paper - based sensors with other sensors. Radar chart compares the manufacturability and performance of CF/GO/Cellulose paper-based electrochemical sensor with sensors from other studies ${ }^{23,31-37}$

useful characteristics: it can be patterned using simple cutting operations, it is flexible and stable in water, and its electrocatalytic activities enable sensitive and selective chemical analysis. We envision that these characteristics of the composite provide the opportunity to realize the dream for portable, low-cost, and sustainable paper-based electrochemical detection.

\section{METHODS}

\section{Materials}

Graphene oxide (GO) (piece of diameter $20 \mu \mathrm{m}$, thickness $<5 \mathrm{~nm}$ ) was purchased from Xiwang Company (Shanghai, China). Cellulose pulp (Poplar chemical pulp) was purchased from Huatai Paper Industry Co., Ltd (Dongying, China). Carbon fiber (CF) (diameter 6 um, length $4.5 \mathrm{~mm}$ ) was purchased from Shenzhen Yataida Science \& Technology Co. Ltd (Shenzhen, China). Cationic polyacrylamide (CPAM) was purchased from Tianjin Zhiyuan Chemical Reagent Co. Ltd (Tianjin, China). Hydroquinone $(\mathrm{HQ})$, p-chlorophenol (CP), p-nitrophenol (NP), and other chemicals were purchased from Sigma-Aldrich. All reagents were used as received without further purification. Ultrapure water $(>18 \mathrm{M} \Omega$ ) prepared by Super Milli-Q water system was used for experiments.

\section{Preparation of CF/GO/cellulose paper}

A $0.3 \%$ GO slurry in water was obtained after $1 \mathrm{~h}$ of ultrasonic treatment with a cell crusher. Preparation of mixed pulp: softwood pulp board $(1 \mathrm{~g})$, which is $99 \%$ cellulose, was dispersed into $1 \mathrm{~L}$ of water, after which a specific amount of the GO slurry was added and stirred evenly, and then a specific amount of CPAM was added and stirred until the water became clear. CF $(1 \mathrm{~g})$ was dispersed in $100 \mathrm{ml}$ of water and then poured into the freshly prepared $\mathrm{GO} /$ cellulose slurry and mixed well. Finally, this mixed pulp was poured into the Kaiser paper making machine (RK3AKWT, Austria) and diluted with $5 \mathrm{~L}$ of water, and then make the $\mathrm{CF} / \mathrm{GO} /$ Cellulose paper. Although the concentrations varied between experiments a typical pulp was composed of $45.9 \%$ cellulose, $45.9 \% \mathrm{CF}, 8 \% \mathrm{GO}$, and $0.2 \%$ CPAM. All test samples were prepared in the laboratory; scalable fabrication of composite paper (Fig. 1a) is prepared by a pilot-scale paper machine in the same proportion using $50 \mathrm{~kg}$ of softwood pulp.

\section{Electrochemical reduction of GO}

$\mathrm{GO}$ of the $\mathrm{CF} / \mathrm{GO} /$ cellulose paper was electrochemically reduced using a three-electrode system, in which the counter electrode was a platinum plate, the reference electrode was $\mathrm{Ag} / \mathrm{AgCl}$, the electrolyte was $1 \mathrm{M} \mathrm{KCl}$ solution, the reaction condition was $-0.9 \mathrm{~V}$, and the reaction time was varied, depending on the experiment 100-1000 s.

\section{Electrochemical measurements}

Cyclic voltammograms (CV) were performed using a three-electrode system (platinum counter electrode and a $\mathrm{Ag} / \mathrm{AgCl}$ reference electrode), a scan rate of $100 \mathrm{mV} \mathrm{s}^{-1}$, and a potential range between $-0.2 \mathrm{~V}$ and $0.7 \mathrm{~V}$. and a $1 \mathrm{M} \mathrm{KCl}$ electrolyte containing $5 \mathrm{mM} \mathrm{K}_{3} \mathrm{Fe}(\mathrm{CN})_{6}$. Electrochemicalimpedance spectroscopy was performed using $5 \mathrm{mM} \mathrm{K}_{3} \mathrm{Fe}(\mathrm{CN})_{6} / \mathrm{K}_{4} \mathrm{Fe}(\mathrm{CN})_{6}$ and a frequency range of $100 \mathrm{kHz}$ to $0.01 \mathrm{~Hz}$.

\section{Electrochemical sensing}

The phenols $\mathrm{HQ}, \mathrm{CP}$, and NP were dissolved in $0.1 \mathrm{M}$ phosphate buffer $(\mathrm{pH} 7)$ and then detected using composite paper as the working electrode in a three-electrode system. Measurements were performed using differential pulse voltammetry (DPV) with the potential range of $0-1.1 \mathrm{~V}$.

\section{DATA AVAILABILITY}

All relevant data that support the findings of this study are available from authors upon reasonable request.

Received: 12 October 2021; Accepted: 6 January 2022; Published online: 14 February 2022

\section{REFERENCES}

1. Liu, X. et al. A universal paper-based electrochemical sensor for zero-background assay of diverse biomarkers. ACS Appl. Mater. Interfaces 11, 15381-15388 (2019).

2. Nonaka, L. H., Almeida, T., Aquino, C. B., Domingues, S. H. \& Souza, V. Crumpled graphene decorated with manganese ferrite nanoparticles for hydrogen peroxide sensing and electrochemical supercapacitors. ACS Appl. Nano Mater. 3, 4859-4869 (2020).

3. Hafez, A. M., Sheng, J., Cao, D., Chen, Y. \& Zhu, H. Flexible lithium metal anode featuring ultrahigh current density stability with uniform deposition and dissolution. ES Energy Environ. 5, 85-93 (2019).

4. Wang, Y., Luo, J. \& Liu, J. Label-free microfluidic paper-based electrochemical aptasensor for ultrasensitive and simultaneous multiplexed detection of cancer biomarkers. Biosens. Bioelectron. 136, 84-90 (2019).

5. Sakar, M., Chandan, H. R. \& Shwetharani, R. Graphene paper-based electrochemical sensors for biomolecules. Graphene Based Electrochem. Sens. Biomol. 1 297-320 (2019)

6. Liu, H., Pang, B. \& Tang, Q. Self-assembly of surface-acylated cellulose nanowhiskers and graphene oxide for multiresponsive Janus-like films with timedependent dry-state structures. Small 16, 1-10 (2020).

7. Li, T., Chen, C. \& Alexandra, H. B. Developing fibrillated cellulose as a sustainable technological material. Nature 590, 47-56 (2021).

8. Wang, P. et al. Development of a paper-based, inexpensive, and disposable electrochemical sensing platform for nitrite detection. Electrochem. Commun. 81, 74-78 (2017).

9. Yakoh, A., Chaiyo, S., Siangproh, W. \& Chailapakul, O. A 3D capillary-driven paperbased sequential microfluidic device for electrochemical sensing applications. ACS Sens. 4, 1211-1221 (2019).

10. Cinti, S., Lellis, B. D., Moscone, D. \& Arduini, F. Sustainable monitoring of $\mathrm{Zn}(\mathrm{III})$ in biological fluids using office paper. Sens. Actuators B Chem. 253, 1199-1206 (2017).

11. Yao, B., Yuan, L., Xu, X., Zhang, J. \& Chen, W. Paper-based solid-state supercapacitors with pencil-drawing graphite/polyaniline networks hybrid electrodes. Nano Energy 2, 1071-1078 (2013).

12. Wang, F., Fu, C., Huang, C., Li, N. \& Yu, J. Paper-based closed Au-bipolar electrode electrochemiluminescence sensing platform for the detection of miRNA-155. Biosens. Bioelectron. 150, 111917 (2019).

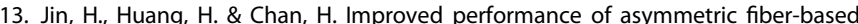
micro-supercapacitors using carbon nanoparticles for flexible energy storage. $J$. Mater. Chem. A 3, 15633-15641 (2015).

14. Han, Y., Zhao, J., Qiu, M., Sun, P. \& Han, D. Hierarchical bi-continuous Pt decorated nanoporous Au-Sn alloy on carbon fiber paper for ascorbic acid, dopamine and uric acid simultaneous sensing. Biosens. Bioelectron. 124-125 191-198 (2019). 
15. Ippolito, S. J., Kandasamy, S., Kalantar-Zadeh, K. \& Wlodarski, W. Hydrogen sensing characteristics of WO3 thin film conductometric sensors activated by Pt and Au catalysts. Sens. Actuators B Chem. 108, 154-158 (2005).

16. Yao, Y., Lan, L., Liu, X., Ying, Y. \& Ping, J. Spontaneous growth and regulation of noble metal nanoparticles on flexible biomimetic MXene paper for bioelectronics. Biosens. Bioelectron. 148, 111799 (2019).

17. Mai, V. P., Ku, C. H. \& Yang, R. Porosity estimation using electric current measurements for paper-based microfluidics. Microfluid. Nanofluidics 23, 59 (2019).

18. Lee, D. J. \& Kim, D. Y. Hydrophobic paper-based SERS sensor using gold nanoparticles arranged on graphene oxide flakes. Sensors 19, 5471 (2019).

19. Fu, H., Song, P., Wu, Q., Zhao, C. \& Liu, X. A paper-based microfluidic platform with shape-memory-polymer-actuated fluid valves for automated multi-step immunoassays. Microsyst. Nanoeng. 5, 50 (2019).

20. Wang, Q., Liu, S., Yang, G., Chen, J. \& Ni, Y. Cationic polyacrylamide enhancing cellulase treatment efficiency of hardwood kraft-based dissolving pulp. Bioresour. Technol. 183, 42-46 (2015).

21. Eduardo, A., Takara, S. V. \& Pereira, M. L. Novel electrochemical sensing platform based on a nanocomposite of PVA/PVP/RGO applied to IgG anti- Toxoplasma gondii antibodies quantitation. Talanta 195, 699-705 (2019).

22. Jalal, N. R., Madrakian, T., Afkhami, A. \& Ghoorchian, A. In situ growth of metal-organic framework HKUST-1 on graphene oxide nanoribbons with high electrochemical sensing performance in imatinib determination. ACS Appl. Mater. Interfaces 12, 4859-4869 (2020).

23. Li, X., Shen, J., Wu, C. \& Wu, K. Ball-Mill-Exfoliated graphene: tunable electrochemistry and phenol sensing. Small 15, 1805567 (2019).

24. Pw, A., Jian, S. A., Kw, A. \& Ny, B. Defect-dependent electrochemistry of exfoliated graphene layers. Carbon 154, 125-131 (2019).

25. Shukla, R. P., Cazelles, R., Kelly, D. L. \& Ben-Yoav, H. A reduced-graphene oxidemodified microelectrode for a repeatable detection of antipsychotic clozapine using microliters-volumes of whole blood. Talanta 209, 120560 (2020).

26. Randles, J. A cathode ray polarograph. Part II.-The current-voltage curves. Trans. Faraday Soc. 44, 327 (1948).

27. Zhou, M., Zhai, Y. \& Dong, S. Electrochemical sensing and biosensing platform based on chemically reduced graphene oxide. Anal. Chem. 81, 5603-5613 (2009).

28. Shamkhalichenar, H. \& Choi, J. W. Review—non-enzymatic hydrogen peroxide electrochemical sensors based on reduced graphene oxide. J. Electrochem. Soc. 167, 37531 (2020).

29. M. Ej Ri, A., Mars, A., Elfil, H. \& Hamzaoui, A. H. Reduced graphene oxide nanosheets modified with nickel disulfide and curcumin nanoparticles for nonenzymatic electrochemical sensing of methyl parathion and 4-nitrophenol. Microchim. Acta 186, 704 (2019).

30. Tsksa, B. \& Kyhab, C. Rational design and preparation of copper vanadate anchored on sulfur doped reduced graphene oxide nanocomposite for electrochemical sensing of antiandrogen drug nilutamide using flexible electrodes. $J$. Hazard. Mater. 410, 124659 (2020).

31. Huo, Z., Zhou, Y. \& Qin, L. Sensitive simultaneous determination of catechol and hydroquinone using a gold electrode modified with carbon nanofibers and gold nanoparticles. Microchim. Acta 173, 119-125 (2011).

32. Du, H., Ye, J., Zhang, J., Huang, X. \& Yu, C. A voltammetric sensor based on graphene-modified electrode for simultaneous determination of catechol and hydroquinone. J. Electroanal. Chem. 650, 209-213 (2011).

33. Wang, Z., Li, S. \& Lv, Q. Simultaneous determination of dihydroxybenzene isomers at single-wall carbon nanotube electrode. Sens. Actuators B Chem. 127, 420-425 (2007).

34. Ding, Y. P., Liu, W. L., Wu, Q. S. \& Wang, X. G. Direct simultaneous determination of dihydroxybenzene isomers at C-nanotube-modified electrodes by derivative voltammetry. J. Electroanal. Chem. 575, 275-280 (2005).
35. Ma, X. M., Liu, Z. N., Qiu, C. C., Chen, T. \& Ma, H. Y. Simultaneous determination of hydroquinone and catechol based on glassy carbon electrode modified with gold-graphene nanocomposite. Microchim. Acta 180, 461-468 (2013).

36. Fan, L., Li, X. \& Kan, X. Disposable graphite paper based sensor for sensitive simultaneous determination of hydroquinone and catechol. Electrochim. Acta 213, 504-511 (2016).

37. González-Costas, J. M., Gómez-Fernández, S., García, J. \& González-Romero, E. Screen-printed electrodes-based technology: environmental application to real time monitoring of phenolic degradation by phytoremediation with horseradish roots. Sci. Total Environ. 744, 140782 (2020).

\section{ACKNOWLEDGEMENTS}

This work was supported by the National Key Research and Development Program of China (2019YFE0114400), the Taishan Industrial Leading Talent Project (20180215), and the United States National Science Foundation (CBET-1932963).

\section{AUTHOR CONTRIBUTIONS}

X.W. and C.W. conceived and designed the work. C.W. prepared paper electrodes and carried out characterization and electrochemical sensing detection. R.W. and H.L. optimized and repeated the experiment. Z.L., W.H., X.S., and G.P. analyzed and interpreted the data. All authors discussed the results and commented on the final paper. X.W. and G.P. supervised the project.

\section{COMPETING INTERESTS}

The authors declare no competing interests.

\section{ADDITIONAL INFORMATION}

Supplementary information The online version contains supplementary material available at https://doi.org/10.1038/s41528-022-00143-1.

Correspondence and requests for materials should be addressed to Xiaohui Wang.

Reprints and permission information is available at http://www.nature.com/ reprints

Publisher's note Springer Nature remains neutral with regard to jurisdictional claims in published maps and institutional affiliations.

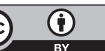

Open Access This article is licensed under a Creative Commons Attribution 4.0 International License, which permits use, sharing, adaptation, distribution and reproduction in any medium or format, as long as you give appropriate credit to the original author(s) and the source, provide a link to the Creative Commons license, and indicate if changes were made. The images or other third party material in this article are included in the article's Creative Commons license, unless indicated otherwise in a credit line to the material. If material is not included in the article's Creative Commons license and your intended use is not permitted by statutory regulation or exceeds the permitted use, you will need to obtain permission directly from the copyright holder. To view a copy of this license, visit http://creativecommons. org/licenses/by/4.0/.

(c) The Author(s) 2022 